\title{
Exponential Capillary Pressure Functions in Sedimentary Rocks
}

\author{
Armin Afrough 1,*, Mehdi Bahari Moghaddam 2, Laura Romero-Zerón 1, Bruce J. Balcom 1 \\ 1University of New Brunswick, Fredericton, Canada \\ 2Petroleum University of Technology, Ahvaz, Iran
}

\begin{abstract}
The Brooks-Corey power-law capillary pressure model is commonly imposed on core analysis data without verifying the validity of its underlying assumptions. The Brooks-Corey model, originally developed to model the pressure head during the drainage of soil, is only valid at low wetting phase saturations. However, such models are often applied in petroleum production simulations and may lead to erroneous recovery factors when the saturation range of interest is far from the end points. We demonstrate that exponential models work much better for capillary pressure compared to the Brooks-Corey model over a wide saturation range. Mercury injection porosimetry, petrographic image analysis, and magnetic resonance studies suggest that the pore and throat size distribution in many rocks are log-normally distributed. This fact was previously employed to calculate the capillary pressure function as a function of saturation for pore size distributions described by a truncated log-normal distribution. Employing a Taylor series expansion, we simplify the random fractal capillary pressure model of Hunt to $P_{c}=\exp (a-b S)$, where $S$ is the wetting phase saturation, and $a$ and $b$ characteristic of the porous medium. An extensive dataset of seventeen centrifuge capillary pressure measurements were used in this research to demonstrate the merit of the new method. For both sandstones and carbonates, the logarithm of capillary pressure showed a linear relationship with saturation as observed by magnetic resonance imaging centrifuge capillary pressure measurements over a wide saturation range. This work demonstrates that: (a) in semi-log plots of capillary pressure as a function of saturation, capillary pressure will vary linearly over a wide saturation range, (b) such a plot as described in (a) will show the uni- or bimodal pore size distribution of the rock, (c) the exponential capillary pressure function simplifies analytical models that use the capillary pressure function, for example oil recovery models for fractured reservoirs.
\end{abstract}

\section{Introduction}

Empirical correlations help establish functional relationships between capillary pressure $P_{c}$ and wetting phase saturation $S_{w}$ in natural porous materials. The Brooks-Corey capillary pressure model [1], the most well-known such equation, reduces the functional relationship between capillary pressure and effective saturation $S_{w e}$ to the bubbling pressure $P_{b}$ and pore-sizedistribution index $\lambda$ according to

$$
P_{c}\left(S_{e}\right)=P_{b} S_{w e}^{-1 / \lambda}
$$

where $P_{b}$ is a measure of the maximum pore size which forms a continuous flow network and $\lambda$ characterizes the pore size distribution. Effective saturation normalizes the wetting phase saturation $S_{w}$ in the range of end-point saturations (residual wetting phase saturation $S_{w r}$ and residual non-wetting phase saturation $S_{n w r}$ ) such that

$$
S_{w e}=\frac{S_{w}-S_{w r}}{\left(1-S_{n w r}\right)-S_{w r}} \text {. (2) }
$$

For primary drainage, with $S_{n w r}=0$, equation (2) reduces to

$$
S_{w e}=\frac{S_{w}-S_{w r}}{1-S_{w r}}
$$

Brooks and Corey developed the above relationship for $S_{w}>S_{w r}$, although saturations less than $S_{w r}$ can exist. This is because $S_{w r}$, as calculated with the method outlined in [1], is an interpolation of irreducible wettingphase saturation rather than the lowest saturation measured during an experiment. They also acknowledged the effect of hysteresis on capillary pressure values. Other assumptions inherent in this empirical model are that the porous material is isotropic and is undergoing through drainage [1]. Wells and Amaefule [2] and Lekia and Evans [3] demonstrated that the Brooks-Corey capillary pressure model fails for tight gas sand samples. Lekia and Evans [3] circumvented the shortcomings of the Brooks-Corey model by explicitly using the wettingphase saturation, in contrast with the effective saturation. Later, recognition of the applicability of fractal theories to sandstones by Katz and Thompson [4] led to the development of power-law analytical equations for capillary pressure for low wetting phase saturations by Toledo et al. [5]. Yang et al. [6] provides the analytical

* corresponding author: Armin.Afrough@unb.ca

() The Authors, published by EDP Sciences. This is an open access article distributed under the terms of the Creative Commons Attribution License 4.0 (http://creativecommons.org/licenses/by/4.0/). 
derivation of Brooks-Corey capillary pressure function according to the mathematics of fractals. Novy et al. [7] had previously demonstrated that capillary pressure is a power-law function of saturation at low wetting phase saturations by modeling fluid flow in networks and considering the effect of disjoining pressure. The most recent advance in modeling relative permeability and capillary pressure in porous materials, developed by Hunt [8], will be reviewed and examined in this work. The main goal of this work is to demonstrate that exponential capillary pressure functions are superior to the model of Brooks-Cory over a wider wetting-phase saturation range. In this paper we show first that the capillary pressure model of Hunt is approximately exponential, even for $D<3$. This is confirmed with an extensive experimental data set taken from the literature. Secondly, the effect of bimodal pore size distributions on $P_{c}$ will be discussed. Finally, we present the application of such models in analytical solutions of gravity drainage in fractured reservoirs.

\section{Theory}

Hunt [8] investigated random fractal models of porous materials applicable to soil systems. They applied critical path analysis in the form of continuum percolation to predict relative permeability. Assuming a finite-range power-law distribution of pore radii, from $r_{0}$ to $r_{m}$, the probability density function for pore radii of a random fractal porous material would be $W(r) \propto r^{-1-D}$. The fractal dimension $D$ of such system of porosity $\phi$ is $[8, \mathrm{p}$. 45]

$$
D=3-\frac{\log (1-\phi)}{\log \left(\frac{d_{m}}{d_{0}}\right)}
$$

The pressure required to remove the wetting phase from a pore of size $r$ is $P_{c}=B / r$, where $B$ is a constant. With a non-wetting entry pressure of $P_{c A}=B / r_{m}$, the wetting phase saturation is [9]

$S_{w}=\left(\frac{3-D}{r_{m}^{3-D}}\right) \int_{r_{0}}^{B / P_{c}} r^{2-D} \mathrm{~d} r=1-\left(\frac{1}{\phi}\right)\left[1-\left(\frac{P_{c}}{P_{c A}}\right)^{D-3}\right]$

Only with a porosity of unity, equation 4 is identical to the Brooks-Corey model. Solution of equation 4 for capillary pressure in terms of saturation gives [9]

$$
P_{c}=P_{c A}\left[\frac{1}{1-\phi\left(1-S_{w}\right)}\right]^{\frac{1}{3-D}}
$$

This equation is valid for $D<3$ whereas values more than 3 are non-physical. For $D=3$, the integral of equation 4 is equal to

$$
P_{c}=P_{c A} \exp \left[-\left(1-S_{w}\right) \ln \left(\frac{r_{m}}{r_{0}}\right)\right]
$$

We recognize that equation 5 can be simplified by a Taylor series expansion. Equation 5 simplifies to

$$
\log P_{c}=\log P_{c A}+\frac{\phi}{3-D}\left(1-S_{w}\right)
$$

which is equivalent to

$$
P_{c}=P_{c A} \exp \left[\frac{\phi}{3-D}\left(1-S_{w}\right)\right]
$$

In the next section, experimental data is tested against this model.

\section{Results and Discussion}

We first present the verification of the exponential capillary pressure function, equation 8 , with experimental data. Deviation from this behavior and possible applications in analytical fluid flow models follows.

\subsection{Exponential Capillary Pressure vs. Experimental Data}

Figure 1 demonstrates a typical oil/water primary drainage capillary pressure in a sandstone core plug as a function of the wetting phase saturation in a semi-log plot. In the saturation range of $(0.23,0.75)$, the exponential capillary pressure function fits experimental data, shown by filled circles, with a coefficient of determination of $R^{2}=0.993$. The range of validity of this descriptive behavior is marked with vertical dashed lines. For $S_{w}>0.75$, experimental capillary pressure data, shown as open squares $\square$, deviate from the exponential $P_{c}$. Deviation from the exponential capillary pressure at high and low saturations is due to fluid flow dominated by the effect of macropores [8] and surface films [5, 7], respectively. Equation 8 was tested with 17

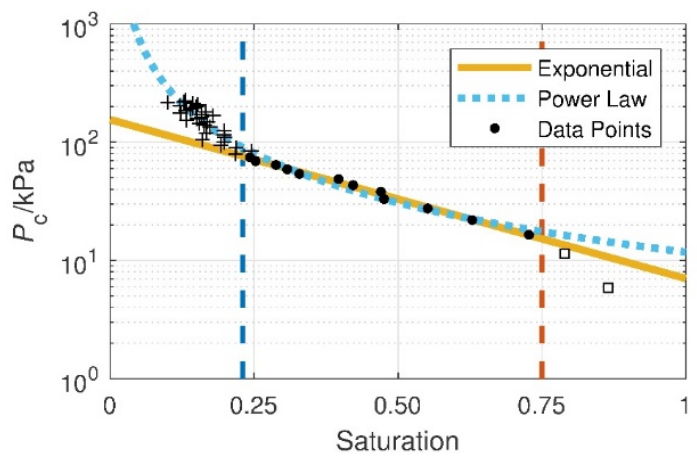

centrifuge capillary pressure measurements on 
consolidated rock core plugs, with water permeabilities in the range of 0.0014 to $0.69(\mu \mathrm{m})^{2}$ and porosities in the range of 0.14 to 0.48 . The experimental datasets are found in the literature in $[1,10,11,12,13,14$, and 15]. Six datasets represent displacement of water by oil; the others are air/water centrifuge experiments. All, but two datasets, [1] and [14], were measured employing saturation profile measurements by either magnetic resonance or nuclear tracer imaging.

Figure 1. A typical oil/water drainage capillary pressure as a function of the wetting phase saturation in a semi-log plot. The exponential capillary pressure function of $\log _{10} P_{c} / \mathrm{kPa}=-1.344 S_{w}+2.191$ fits experimental data $\bullet$ in the saturation range of $(0.23,0.75)$, shown by the vertical lines, with $R^{2}=0.993$. Deviation from this

exponential function is because of film flow + and macropores $\square$ at low and high saturations, respectively.

The Brooks-Corey capillary pressure function $\cdots$ of $P_{c} / \mathrm{kPa}=11.75 S_{w}^{-1.389}$ were obtained by a descriptive fit to experimental data $\bullet$ and + . It appears that the line $\cdots$ fits data well, however, the deviation of data from the fitted line $\cdots$ in the range of $(0.23,0.75)$ demonstrates an obvious trend that is far from random. Data from [10].

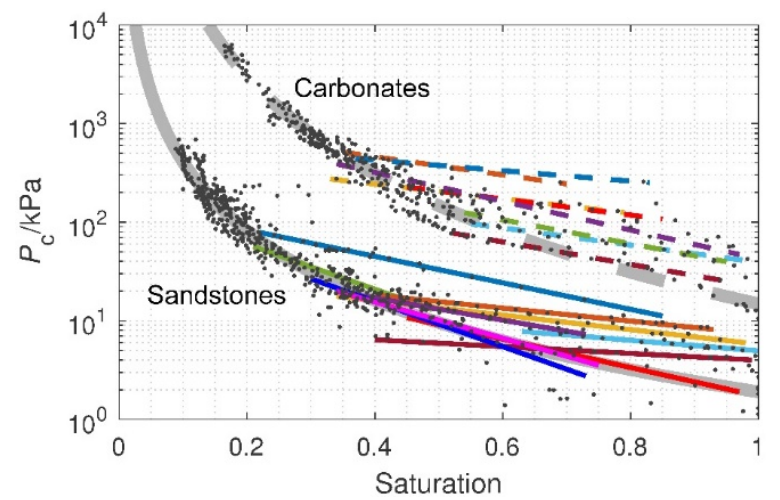

(a)

Semi-log

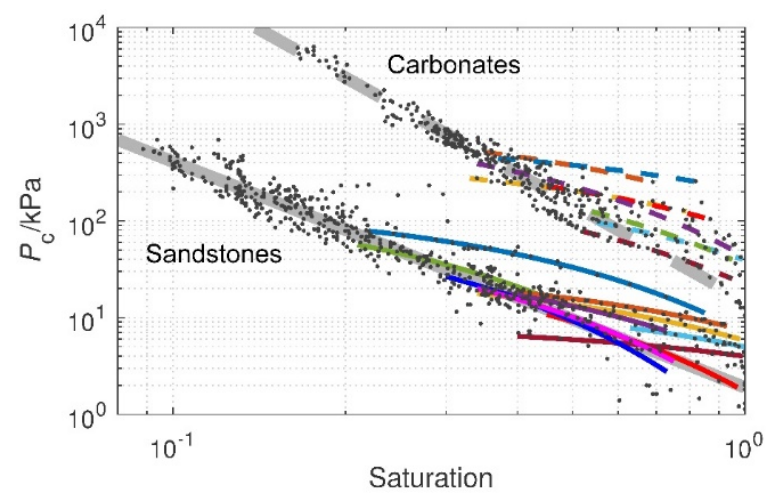

(b)

Log-log

Figure 2. Seventeen experimental datasets of drainage capillary pressure by centrifuge methods shown by $\bullet$. Colored lines represent descriptive fits of exponential capillary pressure functions in the range of their validity to sandstone - and carbonate --- samples. (a) is the $P_{c}-S_{w}$ relationship in a semi-log graph and (b) is the same data in the log-log form. All $P_{c}-S_{w}$ data at low

wetting phase saturations collapse to power-law relationships, one for sandstones and one for carbonates shown by gray lines, representing the conditions at which film flow dominates. The exponential capillary pressure function fits experimental data down to a saturation on the power-law line.

All seventeen capillary pressure datasets employed in this study are shown in Figure 2. The $P_{c}-S_{w}$ relationship is plotted in semi-log and log-log graphs in Figures $2 \mathrm{a}$ and $2 \mathrm{~b}$, respectively. Color lines represent descriptive fits to experimental data points in each dataset. Both sandstones and carbonates are represented in this graph. Data points are not differentiated according to the datasets or the displacing fluid due to the sheer amount of data. The exponential capillary pressure function, equation 8 , only fits a segment of the $P_{c}-S_{w}$ experimental data points; the largest and smallest such range are $\Delta S_{w}=0.6$ and 0.3 , respectively. The average coefficient of determination for the fitted segments is above 0.90 for all data sets, except for four data sets of Baldwin and Yamanashi [13] in which saturation had high uncertainty due to less-quantitative MRI methods. The coefficient of determination was in the range of 0.65 to 0.85 for exponential fits to appropriate segments of data from Baldwin and Yamanashi [15]. Figure 2 demonstrates the advantages of centrifuge capillary pressure methods based on saturation profile measurement over the traditional centrifuge method by providing more data points. All capillary pressure data collapse to a power law relationship at low wetting phase saturations. These power law relationships are shown by straight gray lines of $P_{c} / \mathrm{kPa}=1.9 S_{w}^{-2.33}$ for sandstones and $P_{c} / \mathrm{kPa}=14.86 S_{w}^{-3.3}$ for carbonates in Figure $2 \mathrm{~b}$. This agrees with thin film models at low wetting phase saturation, when the disjoining contribution dominates the capillary contribution [5]. From the data collapse, the fractal dimension of sandstone and carbonate rocks probed by the thin liquid films of water is 2.57 and 2.70, respectively; in agreement with common fractal dimensions measured by Thompson and Katz [3] and Toledo et al. [5] for sedimentary rocks. According to 
Figure 2, in most cases, the Brooks-Corey capillary pressure model under predicts capillary pressure, if fitted to low-saturation $P_{c}\left(S_{w}\right)$ data. Another consequence of inappropriate use of the Brooks-Corey model is that it over-predicts the term $\left(-d P_{c} / d S_{w}\right)$ and hence the capillary pressure dispersion. Any errors in the capillary dispersion term results in erroneous prediction of twophase transition zones in a displacement process. However, note that the Brooks-Corey model correctly predicts $P_{c}$ in the low wetting phase saturation regime.

\subsection{Deviation from the Exponential Capillary Pressure Model}

In the previous subsection, it was demonstrated that capillary pressure in consolidated rocks can be described by an exponential relationship over a wide saturation range. Therefore, semi-log plots of $P_{c}\left(S_{w}\right)$ can be employed for quality control of experimental data. Deviations from this simple relationship can occur because of structural pores, at high saturations, and film flow, at low saturations. An example of these effects is demonstrated in Figure 1. Film flow capillary pressure can be estimated by the Brooks-Corey equation with appropriate parameters.

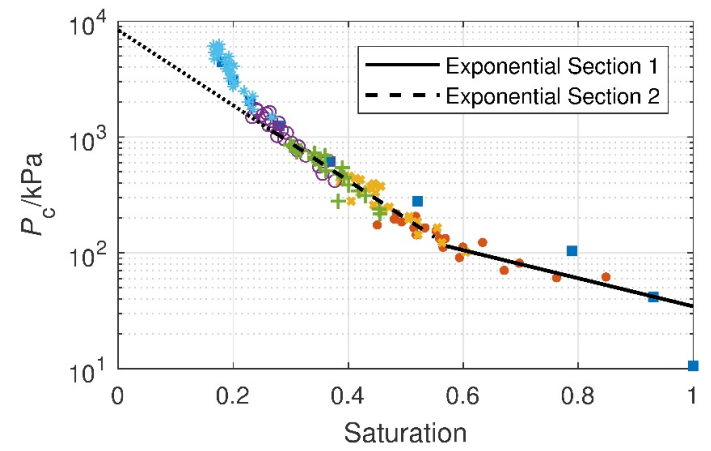

Figure 3. Air/water capillary pressure for carbonate sample E13. Data from a porous plate experiment $\boldsymbol{~ a n d ~ a ~}$ centrifuge capillary pressure measurement of rotation speeds $2800 \bullet, 4000 \times, 5600+, 7900 \circ$, and $15000 *$

revolutions per minute agree each other. Two exponential capillary pressure functions of

$\log _{10} P_{c} / \mathbf{k P a}=-1.213 S_{w}+2.751(-)$ and

$\log _{10} P_{c} / \mathbf{k P a}=-3.248 S_{w}+3.922(--)$ fit the

experimental data in the saturation ranges of

$(0.57,0.94)$ and $(0.27,0.55)$ with $R^{2}=0.90$ and

0.93 , respectively. Deviation from the exponential

function $(\cdots)$ is likely because of film flow at low saturations. Data from [15].
Deviation from the simple exponential function, however, is not limited to the effects described earlier. Hunt and Gee [16], Satyanaga et al. [17], and Zhou et al. [18] previously showed that capillary pressure in soil samples with a bimodal pore size distribution possesses bi-exponential behavior. Such an effect was also observed in this research; notably for sample E13, a carbonate core plug. Figure 3 demonstrates the biexponential capillary pressure function $P_{c}\left(S_{w}\right)$ which follows $\log _{10} P_{c} / \mathrm{kPa}=-1.213 S_{w}+2.751$ in the saturation range of $(0.57,0.94)$ and $\log _{10} P_{c} / \mathrm{kPa}=-3.248 S_{w}+3.922$ in the saturation range of $(0.27,0.55)$. At low saturations, capillary pressure data deviate from the exponential fit due to film flow. This bi-exponential feature is likely due to heterogeneities in the carbonate rock sample E13.

\subsection{Application in Analytical Solutions of Oil Recovery from Fractured Reservoirs}

Consider gravity drainage in a one-dimensional matrix block occupied by oil, as the wetting phase, and gas. At any point in the matrix, the flow rate of the oil phase can be estimated by [19]

$$
q=\frac{k k_{r o}}{\mu_{o}}\left(\Delta \rho g-\frac{d P_{c}}{d z}\right) .
$$

The absolute value of the derivative of capillary pressure with respect to time $\left(-d P_{c} / d S_{w}\right)$ simplifies $d P_{c} / d z$ to $\frac{d P_{c}}{d z}=\frac{d P_{c}}{d S} \frac{d S_{w}}{d z}$

where $d P_{c} / d S_{w}$ is an exponential function; assuming that the exponential capillary pressure model applies. The critical path analysis in random fractals also results in exponential relative permeability functions which further simplifies equation 9 to yield an exponential recovery factor in time like those previously calculated numerically [20] employed in the emerging fractured reservoir simulation methodology of Mittermeir and Heinemann [21].

\section{Conclusions}

The capillary pressure model of Hunt developed for random fractals with truncated log-normal pore size distributions was simplified to an exponential function by employing a Taylor series expansion. This exponential function was shown to fit experimental data very well. Deviations from the exponential capillary pressure behavior are attributed to the effects of structural pores and film flow at high and low wetting phase saturations, respectively. The possibility of bi-exponential capillary 
pressure functions was demonstrated for a carbonate core plug. Exponential capillary pressure functions, along with their relative permeability counterparts, can simplify oil recovery factors in analytical solutions of flow equations for gravity drainage in fractured reservoirs among many others.

Bruce J. Balcom thanks the Canada Chairs program for a Research Chair in MRI of Materials (2009-2023) and NSERC for a Discovery grant. Armin Afrough thanks the Association of Professional Engineers and Geoscientists of New Brunswick and the New Brunswick Innovation Foundation for an Advanced Studies Scholarship and a Science, Technology, Engineering, and Mathematics Award, respectively. The authors also thank the Atlantic Innovation Fund, ConocoPhillips, Saudi Aramco and Green Imaging Technologies for financial support. UNB Libraries, and in particular Pamela Smith, Beverly Benedict, and Susan Malone from the Science and Forestry Library, are acknowledged for their exceptional assistance.

\section{References}

1. Brooks, R.H., A.T. Corey, Hydrology Papers: Hydraulic Properties of Porous Media, Colorado State University, Fort Collins, (1964), pp. 2-4.

2. Wells, J.D., J.O. Amaefule, " Capillary Pressure and Permeability Relationships in Tight Gas Sands," SPE-13879-MS presented at the SPE/DOE Low Permeability Gas Reservoirs Symposium, Denver, Colorado, U.S.A., 19-22 March (1985).

3. Lekia, S.D.L., R.D. Evans, "A Water-Gas Relative Permeability Relationship for Tight Gas Sand Reservoirs," Journal of Energy Resources Technology, (1990) 112, 4, 239-245.

4. Thompson, A.H., A.J. Katz, C.E. Krohn, "The Microgeometry and Transport Properties of Sedimentary Rock," Advances in Physics, (1987) 36, 5, 625-694.

5. Toledo, P.G., R.A. Novy, H.T. Davis, L.E. Scriven, "Capillary Pressure, Water Relative Permeability, Electrical Conductivity and Capillary Dispersion Coefficient of Fractal Porous Media at Low Wetting Phase Saturations," SPE Advanced Technology Series, (1994) 2, 1, 136-141.

6. Yang, Y., C. He, F. Lin, "Comments on "Reply to comments on "Analytical Derivation of BrooksCorey Type Capillary Pressure Models Using Fractal Geometry and Evaluation of Rock Heterogeneity" "," Journal of Petroleum Science and Engineering, (2015) 133, 713-715.

7. Novy, R.A., P.G. Toledo, H.T. Davis, L.E. Scriven, "Capillary Dispersion in Porous Media at Low
Wetting Phase Saturations," Chemical Engineering Science, (1989) 44, 9, 1785-1797.

8. Hunt, A.G., Percolation Theory for Flow in Porous Media, Springer-Verlag, Heidelberg, (2004).

9. Hunt, A.G., "An Explicit Derivation of an Exponential Dependence of the Hydraulic Conductivity on Relative Saturation," Advances in Water Resources, (2004) 27, 2, 197-201.

10. Cano Barrita, P.F.de J., B.J. Balcom, M.J. McAloon, D.P. Green, J. Dick, "Capillary pressure measurement on cores by MRI," Journal of Petroleum Technology, (2008) 60, 8, 63-66.

11. Chen, Q., B.J. Balcom, "Measurement of Rock-Core Capillary Pressure Curves using a Single-Speed Centrifuge and One-Dimensional MagneticResonance Imaging," Journal of Chemical Physics, (2005) 122, 21, Article no. 214720.

12. Fern $\varnothing$, M.A., Ø. Bull, P.O. Sukka, A. Graue, "Capillary Pressures by Fluid Saturation Profile Measurements During Centrifuge Rotation," Transport in Porous Media, (2009) 80, 2, 253-267.

13. Nørgaard, J.V., D. Olsen, J. Reffstrup, N. Springer, "Capillary-Pressure Curves for Low-Permeability Chalk Obtained by Nuclear Magnetic Resonance Imaging of Core-Saturation Profiles," SPE Reservoir Evaluation \& Engineering (1999) 2, 2, 141-148.

14. Green, D.P., J. Gardner, B. J. Balcom, M.J. McAloon, P.F. de J. Cano-Barrita, "Comparison Study of Capillary Pressure Curves Obtained Using Traditional Centrifuge and Magnetic Resonance Imaging Techniques," SPE 110518 presented at the SPE/DOE Improved Oil Recovery Symposium, Tulsa, Oklahoma, U.S.A., 19-23 April (2008).

15. Baldwin, B.A., W.S. Yamanashi, "Capillary-Pressure Determinations from NMR Images of Centrifuged Core Plugs: Berea Sandstone," The Log Analyst (1991) 32, 5, SPWLA-1991-v32n5a6.

16. Hunt, A.G., G.W. Gee, "Application of Critical Path Analysis to Fractal Porous Media: Comparison with Examples from the Hanford Site," Advances in Water Resources, (2002) 25, 2, 129-146.

17. Satyanaga, A., H. Rahardjo, E.-C. Leong, J.-Y. Wang, "Water Characteristic Curve of Soil with Bimodal Grain-Size Distribution," Computers and Geotechnics (2013) 48, (March), 51-61.

18. Zhou, H., S. J. Mooney, X. Peng, "Bimodal Soil Pore Structure Investigated by a Combined Soil Water Retention Curve and X-Ray Computed Tomography Approach," Soil Science Society of America Journal (2017) 81, 6, 1270-1278. 
19. Firoozabadi, A., K. Ishimoto, "Reinfiltration in Fractured Porous Media: Part 1- One Dimensional Model," SPE Advanced Technology Series (1994) 2, 2, 35-44.

20. Pirker, B., G.M. Mittermeir, Z.E. Heinemann, " Numerically Derived Type Curves for Assessing Matrix Recovery Factors," SPE-107074-MS presented at the EUROPEC/EAGE Conference and Exhibition, London, U.K., 11-14 June (2007).

21. Mittermeir, G.M., "Material-Balance Method for Dual-Porosity Reservoirs with Recovery Curves to Model the Matrix/Fracture Transfer," SPE Reservoir Evaluation and Engineering, (2015) 18, $2,171-186$ 